\title{
Ansiedad y depresión identificadas con la Escala de Golberg en estudiantes universitarios del área de la salud
}

Anxiety and Depression Identified in University Students in the Health-care Field with the Goldberg Scale

Ansiedade e depressão identificadas com a escala Goldberg em estudantes universitários da área de saúde

Álvaro Monterrosa-Castro, MD. Esp. ${ }^{1}$ iD, Estéfana Ordosgoitia-Parra. Est ${ }^{2}$ (D) Teresa Beltrán-Barrios. MSc. ${ }^{3}$ (iD

1. Médico. Especialista en Ginecología y Obstetricia. Profesor Titular. Grupo de Investigación Salud de la Mujer. Facultad de Medicina. Universidad de Cartagena. Colombia.

2. Estudiante de Medicina. Grupo de Investigación Salud de la Mujer. Facultad de Medicina. Universidad de Cartagena. Colombia.

3. Filósofa. Maestranda en Filosofía. Grupo de Investigación Salud de la Mujer. Universidad de Cartagena. Colombia.

Correspondencia. Álvaro Monterrosa-Castro. Avenida Venezuela. Edificio City Bank. Oficina-6. Email. alvaromonterrosa@gmail.com

\section{INFORMACIÓN DEL ARTÍCULO:}

Artículo recibido: 03 de Abril 2020

Artículo aceptado: 05 de Agosto de 2020

DOI: https://doi.org/10.29375/01237047.3881

Cómo citar. Monterrosa-Castro A, Ordosgoitia-Parra E, Beltrán-Barrios T, Etal. Ansiedad y depresión identificadas con la Escala de Golberg en estudiantes universitarios del área de la salud. MedUNAB. 2020;23(3):372-388. Doi: https://doi.org/10.29375/01237047.3881

\section{RESUMEN}

Introducción. Los programas universitarios del área de la salud son exigentes y la rutina es extenuante, por lo tanto, generan estrés y un potencial impacto psicológico negativo en los estudiantes. El objetivo de este estudio fue determinar la 
prevalencia y los factores asociados a la ansiedad y a la depresión en estudiantes del área de la salud. Metodología. Estudio de tipo transversal aplicado a estudiantes de Medicina, Enfermería y Química Farmacéutica de una universidad colombiana que diligenciaron anónima y voluntariamente un formulario con datos sociodemográficos y, a su vez, la Escala de Ansiedad y Depresión de Goldberg. Se realizó regresión logística: ansiedad o depresión (variable dependiente) y las características sociodemográficas (independientes); $\mathrm{p}<0.05$, estadísticamente significativo. Resultados. Los participantes corresponden a 697 jóvenes, cuyas edades oscilan entre $20.3 \pm 1.7$. El 43.6\% estudiaba Medicina, el 26.2\% Química Farmacéutica y el 30.1\% Enfermería. Se identificó ansiedad en el 49.8\% (intervalo de confianza 95\%:46.0-53.4) y depresión en el 80.3\% (intervalo de confianza 95\%:77.283.1). Estudiar Medicina, Enfermería, ser de sexo femenino, padecer de sobrepeso e infrapeso, haber tenido consulta previa psicológica o psiquiátrica, tener medicación permanente y sufrir de alguna enfermedad crónica, se asocia a mayor presencia de ansiedad. Proceder de zonas rurales se asoció a mayor depresión. Discusión. Los hallazgos muestran alta prevalencia de síntomas de ansiedad y depresión en el grupo de jóvenes universitarios del área de la salud. Si bien la escala utilizada no hace diagnóstico, sí sugiere la existencia de manifestaciones patológicas. Factores psicosociales, sobre todo del entorno juvenil, estuvieron asociados. Situaciones similares han sido señaladas por diferentes autores. Conclusiones. Se identificó ansiedad en la mitad de los estudiantes y depresión en ocho de cada diez. Factores inherentes a la vida universitaria, costumbres, hábitos y actividades recreativas se asociaron significativamente.

Palabras clave:

Ansiedad; Depresión; Servicios de salud para estudiantes; Adulto joven; Salud Mental.

\section{ABSTRACT}

Introduction. University programs in the health-care field are challenging and their routines are exhausting. Therefore, they create stress and a potential negative psychological impacts on students. This study's objective was to determine the prevalence and factors associated with anxiety and depression in students in the health-care field. Methodology: A cross-sectional study applied to Medical, Nursing and Pharmaceutical Chemistry students from a Colombian university, who anonymously and voluntarily completed a form with sociodemographic data and the Goldberg Anxiety and Depression Scale. Logistic regression was performed: anxiety or depression (dependent variables) and sociodemographic characteristics (independent variables); $\mathrm{p}<0.05$, statistically significant. Results Six hundred and ninety-seven young adults participated, whose ages ranged between 20.3 \pm 1.7. Forty-three point six percent studied Medicine, 26.2\% Pharmaceutical Chemistry and $30.1 \%$ Nursing. Anxiety was identified in 49.8\% (95\% confidence interval:46.0-53.4) and depression in 80.3\% (95\% confidence interval:77.2-83.1). Studying Medicine, Nursing, being female, overweight and underweight, having previously had a psychological or psychiatric consultation, taking permanent medication and suffering from a chronic disease are all associated with a higher presence of anxiety. Being from a rural area was associated with greater depression. Discussion. The findings show a high prevalence of symptoms of anxiety and depression in the group of university students in the health-care field. Even though the scale that was used does not perform diagnoses, it does suggest the existence of pathological manifestations. Psychosocial factors were associated, especially in juvenile environments. Similar situations have been indicated by various authors. Conclusion. Anxiety was identified in half of the students and depression was identified in eight of every ten. Factors inherent to university life, customs, habits and recreational activities were significantly associated.

Keywords:

Anxiety; Depression; Student Health Services; Young adult; Mental Health.

\section{RESUMO}

Introdução. Os programas universitários da área da saúde são exigentes e a rotina é extenuante, gerando estresse e um potencial impacto psicológico negativo nos estudantes. O objetivo deste estudo foi determinar a prevalência e os fatores associados à ansiedade e à depressão em estudantes da área 
de saúde. Metodologia. Trata-se de um estudo transversal aplicado a estudantes de Medicina, Enfermagem e Química Farmacêutica de uma universidade colombiana que preencheram, de forma anônima e voluntária, um formulário com dados sociodemográficos, assim como a Escala Goldberg de Ansiedade e Depressão. Foi realizada regressão logística: ansiedade ou depressão (variável dependente) e características sociodemográficas (independentes); $\mathrm{p}<0.05$, estatisticamente significativo. Resultados. Os participantes foram 697 jovens, cujas idades variam entre os $20.3 \pm 1.7$ anos. $43.6 \%$ estavam cursando Medicina, 26.2\% Química Farmacêutica e 30.1\% Enfermagem. Foi identificada ansiedade em 49.8\% (IC 95\%:46.0-53.4) e a depressão em 80.3\% (IC 95\%: 77.2-83.1). Estudar Medicina, Enfermagem, ser do sexo feminino, estar com sobrepeso e abaixo do peso, ter feito consulta psicológica ou psiquiátrica anterior, ter medicação permanente e sofrer de alguma doença crônica, são fatores que estão associados a uma maior presença de ansiedade. Vir de áreas rurais foi associado a uma maior depressão. Discussão. Os resultados mostram uma alta prevalência de sintomas de ansiedade e depressão no grupo de jovens universitários da área da saúde. Embora a escala utilizada não faça um diagnóstico, sugere a existência de manifestações patológicas. Fatores psicossociais, especialmente no ambiente juvenil, foram associados. Situações semelhantes têm sido apontadas por diferentes autores. Conclusão. A ansiedade foi identificada em metade dos estudantes e a depressão em oito de cada dez. Fatores inerentes à vida universitária, costumes, hábitos e atividades recreativas foram significativamente associadas.

Palavras-chave:

Ansiedade; Depressão; Serviço de Saúde do Estudante; Adulto jovem; Saúde Mental.

\section{Introducción}

La prevalencia de los trastornos mentales varía entre 12 y $47 \%$ en los diferentes continentes, y las cifras van en aumento (1). La Organización Mundial de la Salud(OMS) ha señalado que los trastornos mentales constituyen el $10 \%$ de la carga mundial de morbimortalidad, y la economía mundial pierde US \$1 billón anual en productividad debido a la depresión y a la ansiedad (2). Los trastornos mentales generan discapacidad e impactan negativamente los ámbitos biológicos y sociales; además, si no se detectan e intervienen oportunamente, conducen a pérdidas considerables en términos de salud (1-3).

Así como sucede a nivel global (2,4,5-7), en Colombia los trastornos de ansiedad y depresión son una carga importante de enfermedad en hombres y mujeres, identificada en estudiantes universitarios $(8,9)$. La Encuesta Colombiana de Salud Mental del 2015 reportó que la mitad de los adolescentes presentaban al menos un síntoma de ansiedad y todos los jóvenes al menos uno de depresión. A su vez, entre adultos de edad mediana, nueve de cada diez tenía síntomas de ansiedad, y todos alguna manifestación de depresión $(8,9)$.

Por lo general, los estudios universitarios coinciden con la adolescencia tardía o la juventud. Diversos autores señalan alta presencia de patologías psiquiátricas en esas etapas vitales, principalmente ansiedad y depresión, con cifras superiores a las de la población general. Diferencias en las frecuencias de ansiedad y depresión han sido observadas en cuanto a género, con mayor presencia entre mujeres (10-12).

El ambiente universitario, nuevo escenario para el joven, conlleva nuevas responsabilidades, reducción del tiempo destinado al ocio o a la recreación y la necesidad de cumplir con las expectativas familiares, con el consiguiente estrés académico y potenciales riesgos psicosociales (13). A continuación, se presentan algunos factores estresores para los universitarios: las tareas académicas y la falta de tiempo para su realización, lo que es definido como sobrecarga académica, los exámenes o evaluaciones de los profesores y el excesivo número de horas diarias que se deben destinar a la academia, entre otros $(3,4)$. Los estudiantes del área de la salud, por condicionantes propios de dichas profesiones, sobre todo por el volumen de conocimiento a adquirir y las destrezas a desarrollar en las prácticas en laboratorio o clínicas, pueden estar sometidos en mayor magnitud a estos estresores, por lo que se puede esperar mayor alteración psicológica. Se ha indicado que pueden llegar a experimentar agotamiento, reducción en el interés al estudio y la autocrítica, así como la pérdida de control en el manejo del entorno familiar y el desenvolvimiento social. Los estudiantes del área de la salud pueden ver comprometida su salud 
mental si no cuentan con los suficientes recursos de afrontamiento, el reconocimiento situacional personal y el acompañamiento docente o institucional $(1,2,4,5,7)$.

Por un lado, Uchida \& Uchida han señalado que la ansiedad y la depresión están entre los factores de riesgo de suicidio y muerte en estudiantes japoneses (13). De otra parte, los medios de comunicación colombianos, sobre todo la radio y la televisión, periódicamente informan casos de suicidio por universitarios, algunos del área de la salud. Esta situación, pareciese que no se debiera presentar en jóvenes que, de una u otra manera, son privilegiados en acceder a uno de los pocos cupos disponibles de educación superior. La información acerca de la ansiedad y la depresión en población universitaria delárea de la salud en el caribe colombiano es insuficiente, e incluso a nivel nacional y latinoamericano, mientras que amplios datos están disponibles en jóvenes de otras culturas o países que están sometidos a diferentes condiciones sociales $(1,4,5-7)$. Es necesario ampliar la información sobre la ansiedad y la depresión en el entorno colombiano, además de confrontarla con datos globales, visibilizar de mejor forma la problemática, concientizar a la sociedad en general y realizar sugerencias a las instituciones de educación superior. El objetivo de este estudio fue determinar la prevalencia de ansiedad y depresión en estudiantes universitarios del área de la salud, así como identificar factores asociados desde una lista de situaciones psicosociales.

\section{Metodología}

Estudio de tipo transversal, perteneciente a la línea de investigación Colectivos Universitarios y al proyecto de investigación Dinámica Psicosocial en Estudiantes Universitarios (DISEU), ambos declarados ante la Universidad de Cartagena, Colombia. Esta Institución pública de Educación Superior Colombiana oferta cuatro programas del área de la Salud (Medicina, Enfermería, Química Farmacéutica y Odontología), que están concentrados en un campus universitario específico. Para responder a la justificación del estudio y por conveniencia, fueron escogidos tres programas académicos, el cuarto no tenía estudiantes disponibles por razones internas al momento del trabajo de campo. Participaron estudiantes matriculados en el segundo periodo académico del año 2018 , de segundo a décimo semestre de las profesiones de Medicina, Enfermería y Química Farmacéutica. No se incluyeron estudiantes de primer semestre por considerar que estaban en fase de adaptación a la vida universitaria.
Los participantes fueron elegidos aleatoriamente en el campus universitario por integrantes del grupo de investigación, quienes cumplieron el rol de encuestadores, (previamente habían sido capacitados para tal fin); ingresaron a las aulas de clases y explicaron los alcances del estudio. Informaron acerca de cuántos participantes se requerían por curso y motivaron al estudiantado a la participación anónima y voluntaria. Los interesados permanecieron en el salón y llenaron los formularios con libertad de tiempo para el diligenciamiento. Al finalizar la prueba se les tomó el peso $(\mathrm{kg})$ con una balanza digital y la estatura corporal (cm) con un tallímetro de pared. El único criterio de exclusión fue dejar incompleto el formulario. Los documentos fueron conservados así: los diligenciados incorrectamente y los incompletos, fueron enumerados y legajados en la carpeta "Descartados"; los correctos fueron enumerados y legajados en la carpeta "Estudio". Un mismo curso pudo ser abordado varias veces para completar el tamaño de la muestra, por lo que se evitó la participación repetida de algún estudiante. Las dos carpetas se conservaron en custodia.

Se establecieron las siguientes definiciones de acuerdo con el estado nutricional según la clasificación de la OMS: infrapeso $\left(<18.50 \mathrm{~kg} / \mathrm{m}^{2}\right)$, peso normal $\left(18.50-24.99 \mathrm{~kg} / \mathrm{m}^{2}\right)$, sobrepeso (25.00-29.99 $\mathrm{kg} /$ $\left.\mathrm{m}^{2}\right)$, y obesidad $\left(\geq 30.00 \mathrm{~kg} / \mathrm{m}^{2}\right)$. En lo que respecta a la edad se consideró lo siguiente: adolescente (menos de veinte años) y adulto (de veinte en adelante). En cuanto al estudio: nivel de estudio básico (segundo a quinto semestre) y avanzado (sexto a décimo semestre). Rendimiento académico regular (promedio acumulado 3.5 o menos), bueno (3.6-4.0) y alto (4.1 en adelante). Asistente a actos religiosos, cine, espectáculos, restaurantes, discotecas, eventos culturales y prácticas deportivas (acudir al menos una vez en el último mes). La estratificación socioeconómica se realizó de acuerdo a las recomendaciones del Departamento Administrativo Nacional de Estadística (DANE) en Colombia.

Se diseñó un formulario de tres partes. La primera solicitó datos sociodemográficos y aspectos sociales generales de la cotidianidad de los jóvenes, tomados a partir de una lluvia de ideas (edad, sexo, procedencia, estado civil, número de hijos, religiosidad, facultad, semestre cursado y promedio académico; actividad laboral, consumo de café, bebidas energizantes u otros estimulantes, recreación mensual en cine, espectáculos, actos culturales, asistencia a restaurantes y prácticas deportivas; horas semanales de sueño y estudio, así como la etnia, definida por autorreconocimiento y características fenotípicas). La segunda parte interrogó sobre antecedentes de salud mental (consulta previa 
a psicología o psiquiatría, patologías crónicas y uso permanente de medicamentos). A su vez, la tercera parte fue la Escala de ansiedad y depresión de Goldberg (EADG), cuestionario validado de 18 ítems que se agrupó en dos subescalas; la primera del uno al nueve, y la segunda del diez al dieciocho, apropiada para aplicar en consulta no psiquiátrica. Cada ítem poseía dos opciones de respuesta: sí (un punto), no (cero puntos). La puntuación total de cada subescala varió de 0-9. Un puntaje de cuatro o más en la primera subescala define la presencia de ansiedad, y dos o más, en la otra subescala, identifica depresión. Entre más alto el puntaje mayor es la posibilidad de presentar las alteraciones mencionadas. La subescala ansiedad tiene sensibilidad del $82 \%$ y valor predictivo positivo de 0.56 ; la subescala depresión tiene sensibilidad del $85 \%$ y valor predictivo positivo de 0.85 . La especificidad encontrada para ambas subescalas es de $91 \%$ (14).

El tamaño de la muestra se estimó con la calculadora online Netquest, nivel de heterogeneidad de 50\%, margen de error $4 \%$ y nivel de confianza de $99 \%$, estratificado por facultad, semestre de estudio y sexo. La prevalencia esperada de ansiedad o depresión no se consideró entre los factores para el cálculo del tamaño de muestra. El boletín estadístico del año 2017 de la Universidad de Cartagena señaló que en ese año estaban matriculados 772 estudiantes en Medicina, 289 en Química Farmacéutica y 468 en Enfermería, para un total de 1,529 estudiantes de los programas del área de la salud, que son considerados el universo de estudio. El tamaño de la muestra fue de 619 estudiantes: 264 Medicina, 166 Química Farmacéutica y 189 Enfermería. Se agregaron 93 (15.0\%) estudiantes teniendo en cuenta la estratificación ya señalada, con el fin de prever formularios incompletos. Se aplicaron 712 formularios.

En el análisis estadístico la información obtenida fue tabulada en una base de datos Microsoft Excel $^{\circledR} 2016$. El análisis se adelantó con Epi-Info-7 (Centers for Disease Control and Prevention, Atlanta, EE. UU). Los datos continuos se expresan en medias con desviación estándar, los categóricos en absolutos y porcentajes e intervalos de confianza al 95\%. Se calculó el estadístico de confiabilidad Alpha de Cronbach para cada subescala, ya que ellas se evalúan por separado. Lo anterior como parte de la validación del constructo en estudiantes universitarios colombianos (datos no presentados).

Se realizó regresión logística no ajustada para estimar asociación entre ansiedad o depresión (variables dependientes) con treinta factores sociodemográficos y psicosociales: facultad o semestre de estudio, rendimiento o nivel académico, grupo etario, sexo, etnia, estudios adicionales, estado civil, tener hijos, religiosidad, área de procedencia, estado nutricional, consumo de café, bebidas energizantes, consulta psicológica o psiquiátrica, asistencia a cine, discoteca, restaurantes, eventos culturales, práctica deportiva, actividad laboral o extracurricular (variables independientes). La asociación se expresó en razón de probabilidades (OR) con un intervalo de confianza (IC95\%). Además, se estimó el coeficiente de correlación de Spearman entre la puntuación de cada subescala de EADG con las variables cuantitativas: horas semanales de sueño y estudio, tasas diarias de café, índice de masa corporal, número de amigos del mismo y diferente sexo, promedio académico acumulado, número de asistencias mensuales a eventos culturales, deportivos o recreativos. La fuerza de correlación o de interdependencia se interpretó según la guía para el uso apropiado del coeficiente de correlación en investigación médica (15): ninguna correlación: 0.00; despreciable: 0.01-0.29; baja: 0.30-0.49; moderada: 0.500.69; alta: 0.70-0.89; muy alta: 0.90-0.99; y perfecta: 1.00. Pudo ser positiva o negativa, con o sin significancia estadística, $\mathrm{p}<0.05$ fue estadísticamente significativo.

La participación fue anónima y voluntaria, con firma de consentimiento informado previo a la aplicación del formulario, de acuerdo con la declaración de Helsinki. Se tuvieron en cuenta normas científicas, técnicas y administrativas para la investigación en salud, establecidas en la Resolución 8430 de 1993 del Ministerio de Salud de la República de Colombia, lo que permite considerar al estudio como investigación con riesgo mínimo (16). No se tomaron muestras biológicas ni se realizó intervención alguna diferente a la medición de estatura y peso corporal. El proyecto de investigación tiene aprobación según Acta No. 052018 del 5 de febrero del 2018 del Comité de ética en investigaciones de la Clínica Santa Cruz de Bocagrande, en Cartagena, Colombia. A su vez, el grupo posee aval institucional de vicerrectoría de investigaciones de la Universidad de Cartagena, Colombia.

\section{Resultados}

De los 712 formularios aplicados, 15 fueron "descartados" (seis de Medicina, tres de Química Farmacéutica, cuatro de Enfermería y dos con facultad de estudios no informada). Se evaluaron 697 estudiantes, 12.6\% por encima del tamaño de la muestra. Del total de la muestra, $276(39.6 \%)$ eran hombres y $421(60.4 \%)$ mujeres, la edad promedio fue de $20.3 \pm 1.7$, e IMC $22.7 \pm 2.9$. El 43.6\% estudiaba Medicina, mientras que el $26.2 \%$ y el $30.1 \%$, estudiaban Química Farmacéutica y 
Enfermería, respectivamente. La mayoría cursaba tercer semestre: 91 (13.0\%) (IC95\%:10.7-15.7), y la minoría décimo: 67 (9.6\%) (IC95\%:7.6-12.0). No se observó diferencia significativa en la distribución por semestres de estudio. Las características sociodemográficas, aspectos psicosociales, costumbres y antecedentes de salud se presentan en la tabla 1.

Tabla 1. Características sociodemográficas, aspectos psicosociales, costumbres y antecedentes de salud.

$\mathrm{n}=697$

$\mathrm{X} \pm \mathrm{SD}$

0

Edad, años

$20.3 \pm 1.7$

Peso, $\mathrm{kg}$

$64.3 \pm 9.7$

Número de hijos

$0.07 \pm 0.3$

Índice de masa corporal, $\mathrm{kg} / \mathrm{m} 2$

$22.7 \pm 2.9$

Promedio académico acumulado

$3.7 \pm 0.27$

Número de cigarrillos diarios fumados en el último mes

$0.3 \pm 1.6$

Número de tasas diarias de café en el último mes

$1.1 \pm 1.4$

Número de botellas de bebidas energizantes diarias en el último mes

$0.22 \pm 0.6$

Número total de amistades actuales

$25.6 \pm 23.9$

Número de amistades del mismo sexo

$12.1 \pm 11.5$

Número de amistades de otro sexo

$13.5 \pm 13.8$

Número de veces que asistió a cine o diversos espectáculos en el último mes

$0.7 \pm 0.4$

Número de veces que asistió a conciertos, bailes o discotecas en el último mes

$0.6 \pm 0.6$

Número de veces que realizó actividades deportivas en el último mes

$1.6 \pm 3.6$

Número de veces que asistió a restaurantes en el último mes

$2.8 \pm 3.3$

Número de veces que participó en eventos culturales en el último mes

$0.1 \pm 0.3$

Número de horas de sueño semanal en el último mes

$21.7 \pm 4.0$

Número de horas de estudio semanal en el último mes

$12.6 \pm 5.8$

n (\%) [IC95\%]

Adolescentes

$248(35.5)[32.1-39.2]$

Adultos

$449(64.4)[60.7-67.8]$

Masculino

276 (39.6) [36.0-43.2]

Femenino

421 (60.4) [56.7-63.9]

Mestizo

520 (74.6) [71.2-77.7]

Afrodescendiente

149 (21.3) [18.4-24.5] 
Estudiante de Medicina 304 (43.6) [39.9-47.3]

Estudiante de Química Farmacéutica 183 (26.2) [23.1-29.6]

Estudiante de Enfermería $210(30.1)$ [26.8-33.6]

Nivel de estudios básicos

$331(47.4)$ [43.8-51.2]

Nivel de estudios avanzados

$366(52.5)$ [48.8-56.1]

Rendimiento académico regular

208 (29.8) [26.5-33.3]

Rendimiento académico bueno

409 (58.6) [54.9-62.2]

Rendimiento académico alto

80 (11.4) [9.3-14.0]

Doce o más horas de estudio por semana en el último mes

$362(51.9)$ [48.2-55.6]

Tener estudios técnicos realizados previamente

79 (11.3) [9.1-13.9]

Tener estudios tecnológicos realizados previamente

29 (4.1) [2.9-5.9]

Cumplir actividad laboral remunerada

$39(5.6)[4.1-7.5]$

Estado civil soltero

640 (91.2) [89.5-93.6]

Con hijos

$37(5.3)[3.8-7.2]$

Procedencia de área urbana

358 (51.3) [47.6-55.0]

Menos de treinta horas de sueño a la semana en el último mes

$666(95.5)$ [93.7-96.8]

Estrato socioeconómico bajo-bajo

93 (13.3) [11.0-16.0]

Estrato socioeconómico bajo

249 (35.7) [32.2-39.3]

Estrato socioeconómico medio-bajo

237 (34.0) [30.5-37.6]

Estrato socioeconómico medio

94 (13.4) [11.1-16.2]

Estrato socioeconómico medio-alto

$20(2.8)[1.87-4.3]$

Estrato socioeconómico alto

$4(0.5)$ [0.22-1.4]

Infrapeso

61 (8.7) [6.8-11.0]

Normopeso

483 (69.3) [65.7-72.6]

Sobrepeso

$146(20.9)[18.0-24-1]$

Obesidad

7 (1.0) [0.4-2.0]

Nunca haber tenido hábito de fumar

$552(79.2)[76.0-82.0]$

Consumir sustancias estimulantes para mejorar el rendimiento en el estudio

$11(1.5)[0.8-2.8]$

Tener relación de noviazgo actualmente

149 (21.3) [18.4-24.5]

Adelantar actividad sexual al menos una vez en el último mes

$414(59.4)$ [55.7-62.9]

Asistencia al menos a un acto religioso en el último mes

464 (66.5) [62.9-69.9]

Asistieron a cine o diversos espectáculos en el último mes

$499(71.5)[68.1-74.8]$ 
Asistieron a eventos culturales (teatro, presentación, libros) en el último mes

$85(12.2)[9.9-14.8]$

Asistieron a restaurantes en el último mes

478 (68.5) [65.0-71.9]

Asistieron a conciertos, bailes o discotecas en el último mes

$340(48.7)[45.0-52.4]$

Practicaron al menos una actividad deportiva en el último mes

$247(35.4)[31.9-39.0]$

Tener actividades o cursos académicos extracurriculares

$72(10.3)[8.2-12.8]$

Antecedente de consulta con psicología en el último año

$76(10.9)[8.80-13.44]$

Antecedente de tratamiento psicológico en el último año

$8(1.1)[0.5-2.2]$

Antecedente de consulta con el psiquiatra en el último año

$12(1.7)[0.9-2.9]$

Antecedente de tratamiento psiquiátrico en el último año

$5(0.7)[0.3-1.6]$

Padecen alguna enfermedad crónica en los últimos dos años

$60(8.6)[6.8-11.0]$

Usan algún medicamento permanentemente en los últimos dos años

$61(8.7)[6.8-11.0]$

Fuente: tabla elaborada por los autores

La ansiedad fue identificada en 347 (49.8\%) (IC95\%:46.053.4) estudiantes de la salud, 190 (62.5\%) (IC95\%:56.967.7) de Medicina, 108 (51.4\%) (IC95\%:44.4-58.3) de Enfermería y 49 (26.7\%) (IC95\%:20.5-33.8) de Química Farmacéutica. Por su parte, la depresión fue definida en 560 (80.3\%) (IC95\%:77.2-83.1) estudiantes del área de la salud, 245 (80.5\%) (IC95\%:75.7-84.6) de Medicina, 158 (75.2\%) (IC95\%:68.8-80.9) de Enfermería y 157 (85.7\%) (IC95\%:79.8-90.5) de Química Farmacéutica.
La mitad de los estudiantes manifestó tener al menos un síntoma de ansiedad o depresión. El síntoma de ansiedad más reconocido fue haber tenido temblores, hormigueos, sudores y diarrea $(65.8 \%)$, mientras que el más informado de depresión fue haber perdido la confianza en sí mismo (66.1\%). Las respuestas afirmativas a los ítems de las dos subescalas se presentan en la tabla 2. El estadístico de confiabilidad $\alpha$ de Cronbach basado en los elementos tipificados para la subescala ansiedad fue de 0.812 y de 0.845 para depresión.

Tabla 2. Presencia de manifestaciones relacionadas con ansiedad y depresión, escala de Ansiedad y Depresión de Goldberg (EADG)

$$
\mathrm{n}=697
$$

n (\%) [IC $95 \%]$

\begin{tabular}{lll}
\hline 1 & Sentirse muy excitado, nervioso o tenso & $391(56.1)$ [52.3-59.7] \\
\hline 2 & Haber estado muy preocupado por algo & $300(43.0)$ [39.4-46.7] \\
\hline 3 & Sentirse muy irritable & $399(57.2)[53.5-60.8]$ \\
\hline 4 & Tener dificultades para relajarse & $403(57.8)[54.1-51.4]$ \\
\hline 5 & Dormido mal, tener dificultades para dormir & $390(55.9)[52.2-59.6]$ \\
\hline 6 & Tener dolores de cabeza o de nuca & $387(55.5)[51.8-59.1]$ \\
\hline 7 & $\begin{array}{l}\text { Tener los siguientes síntomas: temblores, hormigueos, sudores, } \\
\text { diarrea }\end{array}$ & $459(65.8)[62.2-69.2]$ \\
\hline 8 & Estar preocupado por su salud & $425(60.9)[57.3-64.5]$ \\
\hline 9 & Haber tenido alguna dificultad para quedarse dormido & $404(57.9)[54.2-61.5]$
\end{tabular}




\begin{tabular}{lll}
\multicolumn{2}{l}{ Ansiedad $(*)$} & $347(49.8)[46.0-53.4]$ \\
\hline 10 & Sentirse con poca energía & $388(55.6)[51.9-59.3]$ \\
\hline 11 & Haber perdido el interés por las cosas & $456(65.4)[61.8-68.8]$ \\
\hline 12 & Haber perdido la confianza en usted mismo & $461(66.1)[62.5-69.5]$ \\
\hline 13 & Haberse sentido sin esperanzas & $460(66.0)[62.4-69.4]$ \\
\hline 14 & Dificultades para concentrarse & $401(57.5)[53.8-61.1]$ \\
\hline 15 & Haber perdido peso por falta de apetito & $445(63.8)[60.2-67.3]$ \\
\hline 16 & Haber estado despertando demasiado temprano & $409(58.6)[54.9-62.2]$ \\
\hline 17 & Sentirse enlentecido & $420(60.2)[56.5-63.8]$ \\
18 & Cree tener tendencia a encontrarse peor por las mañanas & $436(62.5)[58.9-66.0]$ \\
\hline Depresión $(* *)$ & $560(80.3)[77.2-83.1]$ \\
$(*) \alpha$ Cronbach: 0.812 & \\
$(* *) \alpha$ Cronbach: 0.845 &
\end{tabular}

Fuente: tabla elaborada por los autores

Varios factores se asociaron a mayor o menor presencia de ansiedad. Estudiar Medicina o Enfermería, con respecto a Química Farmacéutica, se asoció con mayor presencia significativa, de igual forma sucedió con padecer enfermedad crónica, usar medicación permanente y tener consulta previa con psicología o psiquiatría. Del mismo modo, ser de sexo femenino, tener sobrepeso o en infrapeso (para este último estado nutricional se obtuvo OR: 3.50 (IC95\%:1.92-6.37)). Por su parte, los factores que se asociaron significativamente con menor presencia de ansiedad fueron: asistencia a actos religiosos, discotecas o restaurantes, realizar prácticas deportivas, estar en estado civil casado o en unión libre, tener hijos, haber tenido actividad sexual, ser afrodescendiente y proceder de áreas rurales. Por lo contrario, solo fueron dos los factores significativamente asociados a mayor presencia de depresión: consumir bebidas energizantes y tener estudios técnicos. A su vez, estudiar en la facultad de Enfermería, cursar nivel de estudios avanzados, cumplir actividades extracurriculares o tener doce o más horas de estudio semanal, se asociaron a menor presencia de depresión. La única variable que se asoció significativamente con incremento de las dos condiciones mentales evaluadas fue el consumo de bebidas energizantes, se observó OR: 1.90 (IC95\%: 1.26-2.86) para ansiedad y OR: 2.64 (IC95\%:1.38-5.08) para depresión. La tabla 3 presenta los valores estimados de asociación no ajustada para las variables que tuvieron significancia estadística, tanto para ansiedad como para depresión.

Tabla 3. Factores asociados a ansiedad y depresión, regresión logística no ajustada

$$
\mathrm{n}=697
$$

$$
\text { OR }[\text { IC95\%] } \quad \mathrm{p}
$$

Ansiedad

Tener antecedente de consulta psiquiátrica

Estudiante de Medicina (con respecto a Q. Farmacéutica)

Padecer alguna enfermedad crónica

$\begin{array}{ll}5.16[1.12-23.73] & <0.05 \\ 4.55[3.05-6.80] & <0.05 \\ 3.66[1.97-6.80] & <0.05\end{array}$


Infrapeso (con respecto a Normopeso)

Estudiante de Enfermería (con respecto a Q. Farmacéutica)

Tener consulta previa con psicología

Usar un medicamento permanentemente

Consumir bebidas energizantes

Sobrepeso (con respecto a normopeso)

Femenino (con respecto a masculino)

Asistencia a conciertos, bailes o discotecas

Asistencia, al menos, a un acto religioso en el último mes

Afrodescendiente (con respecto a mestizo)

Asistencia a restaurantes

Practicar actividades deportivas

Procedentes de áreas rurales

Con hijos

Tener actividad sexual en el último mes

Unión libre (con respecto a soltero)

Casado (con respecto a soltero)

Depresión

Consumir bebidas energizantes

Estudios técnicos (con respecto a ningún otro estudio adicional)

Procedentes de áreas rurales

Doce o más horas de estudio por semana en el último mes

Nivel de estudio avanzado (con respecto estudio básico)

Estudiante de Enfermería (con respecto a Q. farmacéutica)

Tener actividades extracurriculares

\begin{tabular}{|c|c|}
\hline 3.50 [1.92-6.37] & $<0.05$ \\
\hline $2.89[1.89-4.42]$ & $<0.001$ \\
\hline $2.56[1.53-4.28]$ & $<0.001$ \\
\hline $2.21[1.26-3.85]$ & $<0.001$ \\
\hline $1.90[1.26-2.86]$ & $<0.001$ \\
\hline $1.59[1.09-2.31]$ & $<0.05$ \\
\hline $1.38[1.01-1.87]$ & $<0.05$ \\
\hline $0.66[0.49-0.90]$ & $<0.001$ \\
\hline $0.66[0.48-0.90]$ & $<0.05$ \\
\hline $0.63[0.43-0.91]$ & $<0.05$ \\
\hline $0.60[0.43-0.83]$ & $<0.05$ \\
\hline $0.58[0.43-0.80$ & $<0.001$ \\
\hline $0.53[0.39-0.72]$ & $<0.001$ \\
\hline $0.46[0.23-0.94]$ & $<0.05$ \\
\hline $0.44[0.33-0.61]$ & $<0.001$ \\
\hline $0.19[0.07-0.52]$ & $<0.001$ \\
\hline $0.16[0.05-0.48]$ & $<0.001$ \\
\hline $2.64[1.38-5.08]$ & $<0.05$ \\
\hline $2.24[1.01-4.95]$ & $<0.05$ \\
\hline $1.47[1.01-2.15]$ & $<0.05$ \\
\hline $0.60[0.45-0.98]$ & $<0.05$ \\
\hline $0.57[0.30-0.80]$ & $<0.05$ \\
\hline $0.50[0.29-0.84]$ & $<0.05$ \\
\hline $0.47[0.27-0.81]$ & $<0.05$ \\
\hline
\end{tabular}

Fuente: tabla elaborada por los autores

El número de botellas diarias consumidas de bebidas energizantes fue la única variable que se correlacionó positivamente con las puntuaciones de las subescalas de ansiedad y depresión $(\mathrm{p}<0.05)$. Varias situaciones se correlacionaron negativamente con la puntuación en la subescala de ansiedad: el número total de amistades actuales, la cantidad de amistades del otro o del mismo sexo, el total de actividades deportivas realizadas, la asistencia a discotecas, bailes o restaurantes en el último mes, la edad y el número de hijos. A su vez, el número de horas semanales de estudio se correlacionó negativamente con la puntuación de la subescala de depresión. Los coeficientes de correlación que alcanzaron significancia estadística se presentan en la tabla 4. No obstante, la fuerza de correlación en todas las situaciones valoradas fue despreciable, según los parámetros de interpretación utilizados. 
Tabla 4. Factores asociados a depresión y ansiedad, coeficiente de Correlación de Spearman

\begin{tabular}{|c|c|c|}
\hline rho (IC95\%) & & $\mathrm{p}$ \\
\hline \multicolumn{3}{|l|}{ Ansiedad } \\
\hline Botellas diarias de bebidas energizantes & $0.120(0.045$ to 0.192$)$ & $<0.05$ \\
\hline Promedio académico acumulado & $0.075(0.001$ to 0.149$)$ & $<0.05$ \\
\hline Número total de amistades actuales & $-0.232(-0.301$ to -0.161$)$ & $<0.05$ \\
\hline Número de amistades del mismo sexo & $-0.232(-0.301$ to -0.161$)$ & $<0.05$ \\
\hline Número de amistades del otro sexo & $-0.224(-0.293$ to -0.152$)$ & $<0.05$ \\
\hline Número de actividades deportivas realizadas & $-0.121(-0.194$ to -0.047$)$ & $<0.05$ \\
\hline Número de asistencia a discotecas o bailes & $-0.133(-0.206$ to -0.059$)$ & $<0.05$ \\
\hline Número de asistencia a restaurantes & $-0.095(-0.168$ to -0.021$)$ & $<0.05$ \\
\hline Número de hijos & $-0.087(-0.160$ to -0.012$)$ & $<0.05$ \\
\hline Edad & $-0.075(-0.149$ to -0.001$)$ & $<0.05$ \\
\hline \multicolumn{3}{|l|}{ Depresión } \\
\hline Botellas diarias de bebidas energizantes & $0.100(0.026$ to 0.173$)$ & $<0.05$ \\
\hline Número de cigarrillos fumados diariamente & $0.077(0.002$ to 0.151$)$ & $<0.05$ \\
\hline Horas semanales de estudio & $-0.102(-0.175$ to -0.028$)$ & $<0.05$ \\
\hline
\end{tabular}

Fuente: tabla elaborada por los autores

\section{Discusión}

La ansiedad es un sentimiento de aprehensión, preocupación o temor, cuya fuente a menudo es desconocida o inespecífica para el individuo (17). Dicho trastorno estuvo presente en la mitad de los estudiantes del área de la salud y en el $62.5 \%$ de los que estudiaban Medicina. Otros autores han señalado en estudiantes de diversas profesiones de una universidad colombiana, también valorados con EADG, frecuencia del 76.2\% (11). A nivel global, las cifras reportadas son muy diversas. Mahroon, et al., encontraron 51.5\% de ansiedad en estudiantes de Medicina en Bahrein, al evaluarlos con Beck Anxiety Inventory (18). A su vez, Milić, et al., en Croacia, observaron que el $54.5 \%$ de los estudiantes de Medicina y Enfermería experimentaban síntomas de ansiedad (19). Cifras aún inferiores se reportaron en estudiantes de Medicina en China y Brasil, 21.2\% y $19.5 \%$, respectivamente (20-21). La cifra encontrada en estudiantes de Medicina de Etiopía fue cercana al 30\%, al ser evaluados con The hospital anxiety and depression
Scale (17). También se han reportado cifras superiores: $73.0 \%$ en estudiantes egipcios de Medicina (22) y $66.4 \%$ en estudiantes de Medicina y Odontología en Arabia Saudita (23). Diferente es la frecuencia de ansiedad encontrada en estudiantes del área de la salud a nivel global, e incluso en una misma región o comunidad, especialmente por ser diferentes las herramientas que se utilizan para establecer la medición, el tamaño de la muestra utilizada y las variables consideradas. Influyen, además, los factores estresantes académicos y no académicos, el contexto socioeconómico, cultural, familiar y personal $(11,17,22,24)$. Es necesario que cada región geográfica y, tal vez, cada institución de educación superior, tengan entre los indicadores de salud mental de sus estudiantes de los diferentes programas la frecuencia de ansiedad.

No se observó cambio en la frecuencia de identificación de ansiedad al contrastar los semestres de estudio matriculados, con respecto al segundo curso. Brenneisen, et al., tampoco lo observaron (25). Se evitó incluir 
estudiantes de primer semestre para tener mayor homogeneidad de la población a valorar, ya que se ha señalado que los estudiantes de primer curso se deben adaptar a la transición de la escuela a la universidad, al distanciamiento de sus familiares, a la necesidad de interactuar con un nuevo ambiente de aprendizaje y a las nuevas relaciones interpersonales $(1,17,22-23)$. Se ha señalado que los estudiantes de Medicina de primer año tienen mayor probabilidad de padecer ansiedad que aquellos que están en cursos más avanzados, en Brasil encontraron RR: 3.79 (IC95\%:1.56-9.20), p=0.003 (21) y en Etiopía AOR: 12.06 (IC95\%:2.18-66.72), p<0.05 (17).

Varios autores han indicado que los estudiantes que padecen ansiedad usualmente tienen bajas calificaciones académicas $(22,26)$. Distinto es el panorama en nuestro estudio, el $69 \%$ de los evaluados tenía rendimiento académico bueno o alto, no se observó que los resultados académicos modificaran la frecuencia de ansiedad. El programa académico sí se asoció con cambio en la frecuencia de dicha condición mental. Estudiar Medicina se asoció cuatro veces más a la ansiedad que estudiar Química Farmacéutica. En el mismo sentido, Fawzy y Hamed (22) señalaron que los estudiantes de Medicina tienen más trastornos psicológicos que estudiantes de otras carreras, y agregan que estudiar Medicina puede ser estresante debido a factores académicos (volumen de información, currículo extenso, muchas horas de clases, limitaciones para la recreación, alta frecuencia o elevado nivel de complejidad en los exámenes y la competitividad entre compañeros) y no académicos (expectativas y exigencias familiares, razones financieras y temor al fracaso futuro al no alcanzar las competencias necesarias al ejercer la profesión). Similares afirmaciones realizan Adhikari et al. (27). Los estudiantes del área de la salud de niveles básicos deberían tener en su perfil la capacidad para adaptarse a ambientes exigentes de aprendizaje, a sesiones extensas de prácticas en niveles avanzados y poseer facilidad para interactuar adecuadamente con los entornos de laboratorios o medios hospitalarios expuestos a las vicisitudes de las enfermedades y la muerte. Se ha resaltado la importancia de explorar suficientemente la presencia de ansiedad, como rasgo o como estado, en aspirantes o estudiantes del área de la salud, con el fin de identificar condicionantes que generan consecuencias negativas como el abandono de los estudios, recurrir al uso de diversas sustancias riesgosas con miras a mejorar el rendimiento académico, el establecimiento del síndrome de burnout o incluso, las ideas suicidas $(1,18,27-29)$

A su vez, se observó que ser mujer se asociaba en un $38 \%$ con mayor ansiedad que la presentada en hombres.
Mahroon, et al., (18) reportaron que, en estudiantes de Medicina, las mujeres tenían mayor riesgo de presentar ansiedad, OR: 2.49 (IC95\%:1.37-4.55), p<0.01. Se ha señalado que en ellas predominan algunos trastornos de salud mental debido a factores socioculturales, sobre todo la mayor disposición para admitir los sentimientos ansiosos (11,22). Igualmente, Picco, et al. (1) observaron que las mujeres tienen el doble de probabilidad que los hombres para reconocer los trastornos mentales, poseen mayor conocimiento de los síntomas y son emocionalmente más intuitivas y más competitivas. Es posible que estas cuatro aseveraciones sean argumentos para intentar explicar la mayor angustia o preocupación por los promedios académicos, entre las mujeres (22). En general, entre los factores que predisponen a la mujer a mayor frecuencia de estados de ansiedad, se cuentan: predisposición genética, hormonas sexuales, reactividad endocrina frente al estrés, la mayor biodisponibilidad de algunos neurotransmisores y la coexistencia de determinantes neuropsicológicos $(11,22)$.

Situaciones personales como el estado nutricional o el estado civil, se han identificado como factores asociados a mayor presencia de ansiedad, tal como fue identificado en el estudio. Se ha indicado que los estudiantes universitarios con sobrepeso y obesidad pueden tener menor autoestima, trastorno dismórfico corporal o dismorfofobia, inadecuada percepción de su estado de salud en general, mayor riesgo de estrés psicológico percibido y elevada frecuencia de ansiedad (30). Observamos que estar casado, en unión libre, e incluso tener hijos, se asocia con menor presencia de ansiedad. El apoyo emocional, el acompañamiento, la compresión y la estabilidad, ofrecidas por la pareja o la familia puede ser una circunstancia que favorece la adecuada salud mental de los estudiantes (1). De igual forma, un estudio en universitarios alemanes encontró que estar casado o en relación comprometida protege contra la soledad emocional, definida como la deficiencia de relaciones íntimas y cercanas (31).

En una evaluación a estudiantes de Medicina de una universidad colombiana, se encontró que los estudiantes utilizaban bebidas energizantes para aumentar la tolerancia a muchas horas de estudio y disminuir el tiempo de sueño, con el fin de buscar rendimiento académico de forma más adecuada (32). En el presente estudio, el $16.6 \%$ consumió al menos una botella de bebida energizante diaria en el último mes y, además, observamos que el consumo de esas bebidas se asociaba con un incremento doble de la presencia de ansiedad. Coherentemente, a mayor cantidad de botellas diarias ingeridas fue significativamente mayor la puntuación en ambas subescalas. Richards \& Smith también lo 
han señalado, $\mathrm{y}$ anotan que paradójicamente tales bebidas son utilizadas para levantar el ánimo, creencia que científicamente no está bien fundamentada, aunque contengan altas cargas de cafeína (33). No se observó asociación entre consumo de café y ansiedad; no obstante, se ha reportado que la cafeína precipita, incrementa o ayuda a mantener la ansiedad, razón por la que se sugiere suspender el consumo de café en personas con personalidad ansiosa o bajo estado de ansiedad (22).

Se identificó que tener el antecedente de asistir a consultas psicológicas o psiquiátricas, padecer enfermedades crónicas o tomar medicamentos frecuentemente, se asociaba a mayor presencia de ansiedad. De igual manera, en estudiantes brasileños de Medicina se halló que tomar medicación generaba 2.69 veces mayor probabilidad de presentar ansiedad (21). Un estudio realizado en un gran número de estudiantes de la Universidad de Belgrado indicó haber encontrado asociación entre enfermedades somáticas y alteraciones en la salud mental, especialmente ansiedad, mientras que las actividades recreativas que exploraron se asociaron con menor presencia de dicha condición (34). De igual forma se observa en nuestra evaluación, donde las actividades de recreación y lúdica favorecieron a una menor frecuencia de ansiedad. Evaluaciones amplias como la revisión sistemática de Eime et al., (35) quienes señalaron que varios estudios han propuesto que participar en actividades deportivas competitivas o recreativas, adelantar actividades desescolarizadas o extracurriculares, hacer parte de grupos juveniles o de voluntariados, laborar en trabajos remunerados, recibir clases de música o interactuar en artes escénicas, así como cultivar fundamentación espiritual o religiosa, pueden influenciar positivamente en patrones saludables de comportamiento general y fundamentar en mejor forma el desarrollo personal. Con respecto a la espiritualidad, en el grupo estudiado observamos que la asistencia a actos religiosos en el último mes se asoció con un $40 \%$ de menor frecuencia de ansiedad. Gonçalves, et al., (36) tuvieron similar observación en estudiantes de Medicina y Enfermería. La religiosidad es un sistema de creencias y símbolos compartidos por grupos de personas que fomentan conductas sociales y doctrinales, impulsan el desarrollo de mecanismos adaptativos ante situaciones estresantes, ayudan a afrontar adversidades, ofrecen propósito de vida e incrementan la satisfacción, el bienestar personal y protegen la salud mental (36). Nuestros hallazgos, respaldados por la teorización de varios autores, permiten sugerir que el currículo académico de profesionalización debe involucrar la presencia de actividades recreativas, de esparcimiento y lúdicas, la socialización entre jóvenes con fomento de expresiones corporales, artísticas, cognitivas, deportivas, etc., con miras a reducir el impacto de los estresores académicos y mejorar la salud psicológica y social (34-36).

La depresión es un problema de salud pública que se caracteriza por sentimientos de tristeza, pérdida de energía, desdén por las actividades de interés, baja autoestima y poca concentración $(17,37)$. Se encontró que el $80.3 \%$ de la población estudiada padecía de depresión, sin diferencias de profesión de estudio, mientras que, en Croacia, señalaron que los estudiantes de Medicina tuvieron mayor presencia de depresión que los de Enfermería (19). Nuestras cifras son más elevadas que las informadas en estudiantes de la salud de Arabia Saudita donde encontraron depresión en el 69.9\% (23). También fue más alta que el promedio indicado en la revisión sistemática más amplia disponible, donde se incluyeron 167 estudios transversales y 16 longitudinales, se evaluaron 129.123 estudiantes de medicina de 47 países, donde encontraron prevalencia cruda del 27.2\% (CI95\%: $24.7 \%$ a $29.9 \%$ ), I2 = 98.9\% para síntomas depresivos o depresión (29). Se debe señalar que cada región geográfica tiene sus propias frecuencias de depresión entre los estudiantes universitarios de la salud, con rangos amplios de frecuencia en estudios realizados en un mismo país o en diferentes que poseen similares condiciones socioculturales $(20,38,39)$. Factores diversos influyen para que se observen esas diferencias, especialmente los específicos de los diseños de investigación, instrumentos de evaluación y las variables involucradas, así como la presencia de factores psicobiológicos personales, patrones de crianza familiar y condicionantes sociales ambientales o culturales, que pueden ser elementos de confusión o de interdependencia. No obstante, e independientemente de la magnitud de las cifras, los estudiantes de medicina son entre dos y cinco veces más propensos a experimentar la depresión que la población general de los Estados Unidos, y solo el 15.7\% de los estudiantes de medicina que presentan episodios de depresión acuden a evaluación y terapia profesional (29). Zeng, et al., (20) indican que las exigencias académicas de los estudios profesionales de la salud generan presión y estrés, por tanto, mayor frecuencia de depresión que otros programas universitarios. Esto debe ser tomado en cuenta por las instituciones de educación superior. Además, tener presente que la adolescencia tardía es una fase de cambios físicos, psicológicos y cognitivos que demandan en el joven sólidas estrategias de afrontamiento para consolidar identidad, autonomía y éxito personal, las cuales, en ocasiones, pueden no estar presentes (11). Se debe explorar la existencia de episodios previos de depresión de inicio a edad temprana, ya que suelen hacerse recurrentes y conllevar cuadros de depresión mayor e intentos de suicidio $(23,36)$. A los síntomas de depresión se suma la lentitud 
la insuficiencia económica, las necesidades básicas no satisfechas, la carencia alimentaria, las condiciones de vulnerabilidad o el sometimiento a violencia doméstica y los patrones de afrontamiento para el manejo de las situaciones estresantes de la cotidianidad. En ese sentido, se requieren estudios adicionales en otros grupos estudiantiles universitarios del área de la salud para continuar explorando la problemática.

Se recomienda a las directivas de las instituciones públicas y privadas de educación superior fortalecer y supervisar permanentemente las acciones programadas por las dependencias de bienestar estudiantil. Desde esas instancias se puede reconocer la presencia del deterioro de la salud mental y fomentar las actividades recreativas, prácticas espirituales, programas extracurriculares y dinámicas ocupacionales para enriquecer lúdicamente los espacios de ocio o esparcimiento, puesto que todos son elementos importantes para reducir la ansiedad y mejorar la salud mental $(34-36,40)$. Se recomienda a los docentes todo el empoderamiento necesario para identificar patrones de comportamiento, actitudes y desempeños que puedan sugerir situaciones relacionadas con ansiedad o depresión en sus estudiantes $(2,4,12,18)$. Los profesionales administrativos, e incluso otros estudiantes, pueden ser involucrados en equipos de vigilancia y prevención en cuanto a programas de salud mental (40). Los profesionales de atención primaria, y en general todos los profesionales de la salud que brindan consulta en salud a los estudiantes universitarios, se deben preocupar por identificar tempranamente síntomas de trastornos mentales. Para identificar jóvenes que ameriten ser remitidos a otros espacios clínicos para la valoración, diagnóstico y terapéutica especializada, puede ser útil aplicar en la sala de espera o al interior de la consulta, escalas sencillas y de rápido diligenciamiento como la utilizada en el estudio, o adelantar interrogatorios con términos precisos y exploratorios del bienestar mental.

\section{Conclusión}

Se observó alta prevalencia de ansiedad y depresión en un grupo de estudiantes del área de la salud de una universidad del caribe colombiano. El consumo de bebidas energizantes fue la única variable de un largo número de características sociodemográficas, aspectos psicosociales, costumbres y antecedentes de salud, que se asoció con mayor presencia a la ansiedad y a la depresión. Mientras que tener antecedentes de consulta psiquiátrica, ser estudiante de Medicina o Enfermería, padecer alguna enfermedad crónica, estar en infrapeso o sobrepeso, tener consulta previa con psicología, usar un medicamento permanentemente y pertenecer al sexo femenino, se asociaron con mayor medida a la ansiedad; las 
actividades recreativas como los deportes, los conciertos, los bailes, asistir a discotecas, restaurantes, tener pareja e hijos, hicieron lo contrario. Cursar nivel de estudio avanzado y desarrollar actividades extracurriculares se asociaron con menor frecuencia a la depresión. Los hallazgos del estudio permiten señalar la magnitud de la problemática. Se recomienda a las autoridades sanitarias y educativas la implementación de programas que involucren a todos los estamentos universitarios en la identificación, intervención, prevención y rehabilitación de los escenarios psicosociales que puedan fomentar o agravar la ansiedad y la depresión en los estudiantes del área de la salud.

\section{Agradecimientos}

A los estudiantes de los tres programas del área de la salud de la Universidad seleccionada, que gentilmente dedicaron voluntad y tiempo para aceptar la invitación y diligenciar completamente y con sinceridad el formulario.

\section{Conflicto de interés}

No hay conflictos de interés que declarar.

\section{Financiación}

Los autores no recibieron recursos económicos por su participación en la investigación. El Grupo de Investigación Salud de la Mujer recibió recursos financieros, logísticos y aval de la Universidad de Cartagena para realizar el presente estudio por medio del Plan de Fortalecimiento y Sostenibilidad de Grupos de Investigación. Resolución número 00334-2018 y 014302019.

\section{Referencias}

1. Picco L, Seow E, Chua BY, Mahendran R, Verma S, Chong SA, Subramaniam M. Recognition of mental disorders: findings from a cross-sectional study among medical students in Singapore. BMJ Open. 2017;7(12): e019038. https://doi.org/10.1136/ bmjopen-2017-019038

2. World Health Organization. Mental Health. [internet]. [citado 19 feb de 2020]. Recuperado a partir de: https://www.who.int/news-room/facts-in-pictures/ detail/mental-health

3. Arrieta-Vergara K, Fortich-Mesa N, Tirado-Amador L, Simancas-Pallares M. Common mental disorders and associated factors in dental students from Cartagena, Colombia. Rev Colomb Psiquiatr. 2019;48(1):10-16. https://doi.org/10.1016/j.rcp.2017.05.015
4. Kumar B, Shah MAA, Kumari R, Kumar A, Kumar J, Tahir A. Depression, anxiety, and stress among finalyear medical students. Cureus. 2019;11(3): e4257. https://doi.org/10.7759/cureus.4257

5. Tung YJ, Lo KKH, Ho RCM, Tam WSW. Prevalence of depression among nursing students: A systematic review and meta-analysis. Nurse Educ Today. 2018; 63:119-129. https://doi.org/10.1016/j.nedt.2018.01.009

6. Damasio IL, Pecci N, Kleinsorge R, Granero AL, Cerrato SH, Da Silva O, Lucchetti G. Depression, stress and anxiety in medical students: A cross-sectional comparison between students from different semesters. Rev Assoc Med Bras 2017;63(1):21-28. https://doi. org/10.1590/1806-9282.63.01.21

7. Chatterjee S, Saha I, Mukhopadhyay S, Misra R, Chakraborty A, Bhattacharya A. Depression among nursing students in an Indian government college. $\mathrm{Br}$ J Nurs. 2014;23(6):316-20. https://doi.org/10.12968/ bjon.2014.23.6.316

8. Gómez-Restrepo C, Tamayo-Martínez N, Bohórquez A, Rondón M, Medina-Rico M, Rengifo H, Bautisa N. Depression and anxiety disorders and associated factors in the adult Colombian population, 2015 National Mental Health Survey. Rev Colomb Psiquiatr. 2016;45(Suplement1):58-67. https://doi.org/10.1016/j. rep.2016.04.009

9. MSPS. Ministerio de Salud de Colombia. Guía metodológica para el observatorio nacional de salud mental. [internet]. 2017 [citado 19 de feb 2020]. Recuperado a partir de: https://www.minsalud.gov.co/ sites/rid/Lists/BibliotecaDigital/RIDE/VS/ED/GCFI/ guia-ross-salud-mental.pdf

10. Song Y, Lindquist R. Effects of mindfulness-based stress reduction on depression, anxiety, stress and mindfulness in Korean nursing students. Nurse Educ Today. 2015;35(1):86-90. https://doi.org/10.1016/j. nedt.2014.06.010

11. Arrieta-Vergara K, Díaz-Cárdenas S, GonzálezMartínez F. Síntomas de depresión y ansiedad en jóvenes universitarios: prevalencia y factores relacionados. Rev Clin Med Fam. 2014;7(1):14-22. https://doi.org/10.4321/S1699-695X2014000100003

12. Sarokhani D, Delpisheh A, Veisani Y, Sarokhani MT, Manesh RE, Sayehmiri K. Prevalence of depression among university students: A systematic review and meta-analysis study. Depress Res Treat. 2013; 2013:373-857. https://doi.org/10.1155/2013/373857

13. Uchida $C$, Uchida $M$. Characteristics and risk factors for suicide and deaths among college students: A 23-Year serial prevalence study of data from 8.2 million japanese college students. J Clin Psychiatry. 2017;78(4): e404-e412. https://doi.org/10.4088/ JCP. $16 \mathrm{~m} 10807$

14. Goldberg D, Bridges K, Duncan-Jones P, Grayson D. Detecting anxiety and depression in general medical settings. BMJ. 1988;297(6653):897-899. https://doi. org $/ 10.1136 / \mathrm{bmj} .297 .6653 .897$

15. Mukaka MM. A guide to appropriate use of correlation coefficient in medical research. Malawi Med J. 2012;24(3):69-71. 
multicenter study. BMC Med Educ. 2016; 16(1):282. https://doi.org/10.1186/s12909-016-0791-1

26. AlFaris E, Irfan F, Qureshi R, Naeem N, Alshomrani A, Ponnamperuma G, Al Yousufi N, Al Maflehi N, Al Naami M, Jamal A, van der Vleuten C. Health professions' students have an alarming prevalence of depressive symptoms: exploration of the associated factors. BMC Med Educ. 2016;16(1):279. https://doi. org/10.1186/s12909-016-0794-y

27. Adhikari A, Dutta A, Sapkota S, Chapagain A, Aryal A, Pradhan A. Prevalence of poor mental health among medical students in Nepal: a cross-sectional study. BMC Med Educ. 2017;17(1):232. https://doi. org/10.1186/s12909-017-1083-0

28. Talih F, Daher M, Daou D, Ajaltouni J. Examining Burnout, Depression, and Attitudes Regarding Drug Use Among Lebanese Medical Students During the 4 Years of Medical School. Acad Psychiatry. 2018;42(2):288-296. https://doi.org/10.1007/s40596017-0879-x

29. Rotenstein LS, Ramos MA, Torre M, Segal JB, Peluso MJ, Guille C, Sen S, Mata D. Prevalence of depression, depressive symptoms, and suicidal ideation among medical students: A systematic review and meta-analysis. JAMA. 2016;316(21):2214-2236. https://doi.org/10.1001/jama.2016.17324

30. Phelan SM, Burgess DJ, Puhl R, Dyrbye LN, Dovidio JF, Yeazel M, Ridgeway JL, Nelson D, Perry S, Przedworski JM, Burke SE, Hardeman RR, van Ryn $M$. The Adverse effect of weight stigma on the wellbeing of medical students with overweight or obesity: findings from a national survey. J Gen Intern Med. 2015 Sep;30(9):1251-8. https://doi.org/10.1007/ s11606-015-3266-X

31. Diehl K, Jansen C, Ishchanova K, Hilger-Kolb J. Loneliness at universities: determinants of emotional and social loneliness among students. Int J Environ Res Public Health. 2018;15(9):1865. https://doi. org/10.3390/ijerph15091865

32. Concerto C, Conti C, Muscatello MR, Signorelli MS, Zoccali R, Coira D, Aguglia E, Battaglia F. Sleep quality, perceived stress, and caffeinated drinks intake in psychiatry residents: A cross-sectional Study. J Caffeine Res. 2017;7(1):18-22. https://doi. org/10.1089/jcr.2016.0014

33. Richards G, Smith AP. A review of energy drinks and mental health, with a focus on stress, anxiety, and depression. J Caffeine Res. 2016;6(2):49-63. https:// doi.org/10.1089/jcr.2015.0033

34. Stojanović-Tasić M, Grgurević A, Cvetković J, Grgurević U, Trajković G. Association between somatic diseases and symptoms of depression and anxiety among Belgrade University students. Med Glas (Zenica). 2014;11(2):373-378. 
35. Eime RM, Young JA, Harvey JT, Charity MJ, Payne WR. A systematic review of the psychological and social benefits of participation in sport for children and adolescents: informing development of a conceptual model of health through sport. Int J Behav Nutr Phys Act. 2013; 10:135. https://doi.org/10.1186/14795868-10-135

36. Gonçalves JRL, Jorge AP, Zanetti GC, Amaro EA, Tótoli RT, Lucchetti G. Religiousness is associated with lower levels of anxiety, but not depression, in medical and nursing students. Rev Assoc Med Bras. 2018;64(6):537-542. https://doi.org/10.1590/18069282.64 .06 .537

37. Ngasa SN, Sama CB, Dzekem BS, Nforchu KN, Tindong M, Aroke D, Dimala CA. Prevalence and factors associated with depression among medical students in Cameroon: a cross-sectional study. BMC Psychiatry. 2017;17(1):216. https://doi.org/10.1186/ s12888-017-1382-3
38. Fata Nahas ARM, Elkalmi RM, Al-Shami AM, Elsayed TM. Prevalence of Depression among health sciences students: findings from a public university in Malaysia. J Pharm Bioallied Sci. 2019;11(2):170-175. https://doi.org/10.4103/JPBS.JPBS 263 18

39. Hamasha AA, Kareem YM, Alghamdi MS, Algarni MS, Alahedib KS, Alharbi FA. Risk indicators of depression among medical, dental, nursing, pharmacology, and other medical science students in Saudi Arabia. Int Rev Psychiatry. 2019;31(7-8):646652. https://doi.org/10.1080/09540261.2019.1584095

40. Lemos MA, Henao-Pérez M, López-Medina DC. Stress and mental health in medical students: relation with coping and extracurricular activities. Archivos de medicina. [internet].2018[citado 19 feb de 2020]; 14(2:3):1-8. Recuperado a partir de: file:///C:/Users/ Admin/Downloads/ 